Técnicas de la guitarra clásica aplicadas a la interpretación del bolero

Oscar Julián Alvarado Laurín, Oscar Alejandro Beltrán Sánchez

Epistemus - Revista de estudios en Música, Cognición y Cultura, 8(1), 76-92, e017, 2020

ISSN 1853-0494 | https://doi.org/10.24215/18530494e017

https://revistas.unlp.edu.ar/Epistemus

Sociedad Argentina para las Ciencias Cognitivas de la Música (SACCoM)

Universidad Nacional de La Plata

La Plata | Buenos Aires | Argentina

\title{
Técnicas de la guitarra clásica aplicadas a la interpretación del bolero
}

\author{
Oscar Julián Alvarado Laurín' y Oscar Alejandro \\ Beltrán Sánchez ${ }^{2}$ \\ oalvarado@ucundinamarca.edu.co \\ Universidad de Cundinamarca
}

Resumen

Uno de los objetivos del estudio de un instrumento musical en instituciones académicas es la formación de instrumentistas solistas. La incorporación de géneros tradicionales en dicha formación conlleva la dificultad de adaptar música originalmente ejecutada en conjuntos instrumentales a un instrumento solista. En este artículo se analizan los roles complementarios de las dos guitarras que conforman tradicionalmente el conjunto del bolero y se proponen técnicas instrumentales para que ambas partes puedan ser ejecutadas por una guitarra solista. Las técnicas propuestas son el toque apoyado, el toque tirado, el pizzicato, los armónicos naturales, la ejecución de melodía a dos notas, ligados y apoyaturas, arrastres y uso de barra o ceja. Estas técnicas son aplicadas a seis temas, evaluando en cada caso cuál de ellas se adapta mejor a sus características musicales. El aporte de esta investigación consiste en la aplicación de técnicas instrumentales de la guitarra clásica solista para la ejecución de boleros, fundamental para la incorporación de dicho género en entornos académicos.

\section{Palabras Clave}

Bolero, adaptación, guitarra clásica, música popular, música latina. 


\title{
Contributions of the classic guitar to the interpretation of the bolero
}

\begin{abstract}
One of the objectives of studying a musical instrument in academic institutions is the training of instrumentalists. The incorporation of traditional genres in this level entails the difficulty of adapting music originally performed in instrumental ensembles to a solo instrument. In this article the complementary roles of the two guitars that traditionally make up the bolero ensemble are analyzed to propose instrumental techniques so both parts can be performed by a solo guitar. The proposed techniques are the supported touch, the drawn touch, the pizzicato, the natural harmonics, the execution of a two-note melody, ligatures and supports, dragging and the use of a bar. These techniques are applied to six themes, evaluating in each case which of them best suits their musical characteristics. The contribution of this research consists in the application of instrumental techniques of the classical solo guitar for the performance of boleros, fundamental for the incorporation of this genre in academic settings.
\end{abstract}

\section{Key Words}

Bolero, adaptation, classical guitar, popular music, latin music.

\section{Introducción}

Con motivo de los grandes avances tecnológicos y sociales que se dan a raíz de la globalización, se corre el riesgo de perder la mirada contextualizada de la realidad local al tratar de responder a unas necesidades globales. Específicamente en el campo de las artes musicales, se tiende a priorizar la enseñanza de la música académica como las bases necesarias para la formación musical, ya que es el pilar fundamental de todo desarrollo instrumental y vocal. Sin embargo, en algunos centros de formación musical esta predilección implica negar la posibilidad de reforzar los elementos culturales locales que constituyen uno de los campos laborales más comunes del músico colombiano actual. Teniendo en cuenta que los matices que definen culturalmente a cada sociedad involucran una conjunción entre los elementos ancestrales y los modernos y siendo la música una de las mejores formas de ilustrar la identidad cultural de los pueblos (Buil Tercero y Hormigos Ruiz, 2016), se justifica la exploración de aquellas técnicas propias de 
la guitarra clásica que contribuyen a mejorar la ejecución del intérprete solista en el género bolero.

\section{Recorrido transformativo del género}

El bolero arribó desde Cuba a la Península de Yucatán y conquistó todo México, generando una gran dinámica original y creativa. Se introdujo también con fuerza en Puerto Rico, República Dominicana y Haití, países donde se le ha cultivado con perseverancia y esmero. Su evolución se relaciona con distintos hechos económicos, políticos y culturales ocurridos en América Latina y El Caribe (Villegas, 2016; García, 2016; Arzubiaga, 2007). Ya en Colombia, al interpretar el bolero se eliminaron los tiples y bandolas, instrumentos tradicionales que conforman el trío instrumental típico andino (Londoño y Tobón, 2004). En su lugar, se incorporaron las dos guitarras acompañantes y la guitarra requinto de la tradición boyacense, profundizando en el ritmo de bongó, timbal, maracas y clave para mayor sustentación. Esta adaptación, al fusionarse con elementos culturales propios de cada región colombiana, produjo la aparición de por lo menos tres híbridos dentro del género: el bolero apasillado de Julio Jaramillo Jr., el bolero arrabalero de Alcy Acosta y el bolero guasca o carrilera de Rómulo Caicedo (Pagano, 1989).

$\mathrm{Al}$ interpretar estos géneros y subgéneros de la música popular se concibe a la guitarra de acuerdo a las dos funciones que cumple: guitarra acompañante (o marcante) y guitarra puntera; la primera realiza la armonía y la segunda la melodía. Es así que, para la interpretación del bolero, se suelen acoplar ensambles con dos o tres instrumentos de cuerda, tocando notas y acordes que podrían ser ejecutados por un solo intérprete. En este orden de ideas, el proyecto del cual se desprende este artículo tuvo como principal objetivo la creación de arreglos musicales donde las dos funciones mencionadas sean abordadas por un solo guitarrista a la manera en que se interpreta la guitarra clásica como instrumento solista, convirtiéndose en el protagonista dentro del ensamble musical. Consecuentemente se formuló la pregunta: ¿qué técnicas propias de la música académica para guitarra clásica se pueden utilizar para la interpretación del bolero por parte de un único guitarrista?

\section{Observar el Proceso Desde los Arreglos Musicales}

En este punto se hace necesario contextualizar al lector acerca del entorno en el cual se llevó a cabo la presente investigación. El Programa de Música de la Universidad de Cundinamarca está ubicado en un municipio cercano a Bogotá, la capital del país. La mayoría de sus estudiantes provienen de pueblos aledaños, lo que permite ubicar su realidad dentro de las tradiciones culturales andinas. Esta 
región se caracteriza por la promoción de la música folklórica y tradicional, entendida como aquella que está compuesta por las tradiciones que se heredan a las siguientes generaciones y logran mantenerse en el quehacer de las personas, como parte de su realidad y de su identidad (Thoms, 1846), a través de centros y casas culturales de cada pueblo. Estas instituciones, además, constituyen una de las principales opciones laborales para los futuros músicos, al permitirles desempeñarse como intérpretes, directores o docentes.

Por lo tanto, se plantea la necesidad de abordar dichos ritmos desde una perspectiva académica de forma que permitan desarrollar las competencias requeridas en el currículo, contextualizándolas a las realidades de los estudiantes. Consecuentemente, y de acuerdo con los lineamientos del Plan Rectoral de la Universidad de Cundinamarca 2015-2019, se hace necesario generar nuevos conocimientos en cuanto a la interpretación de la guitarra clásica en el ámbito de las llamadas músicas populares (Barrera, 2015). Es así que el aporte específico del presente proyecto se dirigió hacia el género bolero, buscando relacionar el tema a las inclinaciones musicales de los investigadores.

Entonces, para determinar qué técnicas propias de la música académica para guitarra clásica pueden aportar a la interpretación del bolero por parte de un único guitarrista, se propuso:

- Seleccionar el repertorio a trabajar, teniendo en cuenta que las funciones de los instrumentos se encuentren bien diferenciadas.

- Transcribir y montar los arreglos del repertorio escogido.

- Generar cuatro productos que evidencien el trabajo realizado y sirvan de apoyo educativo para la Universidad.

Consecuentemente, se propendió por la producción de una serie de material documental y audiovisual, para ser utilizado como apoyo educativo a través de diferentes medios de interacción -especialmente la plataforma institucional-, mejorando la calidad de la producción intelectual propuesta por la institución, creando diferentes campos de acción para los guitarristas como parte de los núcleos temáticos de Instrumento principal y Conjuntos, manteniendo los requerimientos técnicos y musicales de su instrumento y brindando la posibilidad de incursionar en la ejecución de ritmos latinoamericanos derivados del bolero.

Finalmente, las acciones ejecutadas en esta investigación responden al proceso creativo de los artistas involucrados durante dos semestres académicos de acuerdo con su propia experiencia profesional, lo que conduce al desarrollo de unas conclusiones particulares más allá de los resultados obtenidos.

Para este proyecto se planteó una metodología cualitativa que, mediante el análisis documental y la observación participante, permitiera analizar e intervenir 
el repertorio escogido y evaluar el alcance de la estrategia planteada. Se trató de una investigación de acción participativa ya que tuvo su base en el análisis de la experiencia obtenida al ejecutar la estrategia propuesta como fuente principal del conocimiento, por lo que priorizó el entendimiento de las relaciones y fenómenos a través de la intervención de los investigadores en el entorno educativo y musical. Según Hugo Cerda (2001), "la investigación-acción participativa es el resultado de una reflexión-investigación continua sobre la realidad abordada, no solo para conocerla, sino para transformarla" (p. 124). Entonces, se buscó generar un impacto positivo en el contexto pedagógico de la institución, así como también en la vida académica y profesional de sus estudiantes.

La población beneficiada de este proyecto son todos aquellos intérpretes de la guitarra, estudiantes y docentes, que precisen adaptar las técnicas de la guitarra clásica al ámbito popular, así como también los investigadores en búsqueda de antecedentes. En este orden de ideas, el estudio se desarrolló en varias fases, descriptas a continuación.

\section{Fase preparatoria}

Se analizó y se escogió la muestra, teniendo en cuenta que las funciones de los instrumentos estuvieran bien diferenciadas y que pertenecieran al género bolero. De las múltiples opciones existentes se seleccionaron seis temas musicales, a saber:

- "Algo contigo". Autor: Bernardo Mitnik (Chico Novarro).

- "Sin ti". Autor: Pepe Guizar.

- "Y". Autor: Mario de Jesús Báez.

- “Sin poderte hablar”. Autor: Willie Colón.

• “Quizás, quizás, quizás". Autor: Osvaldo Farrés.

- "La barca". Autor: Roberto Cantoral.

Dichos temas fueron escogidos cuidadosamente tomando en cuenta la amplia gama de posibilidades técnicas que se puede ejecutar en ellos y el conocimiento previo de las mismas por parte de los investigadores. Su selección se realizó considerando que la interpretación incluyera tanto a la melodía como al acompañamiento, lo que obliga a utilizar al máximo los dedos de la mano derecha y dificulta el uso de pick o plector, tan empleado en la música popular. Estas peculiaridades se observan en mayor medida en introducciones e interludios, donde la guitarra tiene mayor protagonismo, pasando a ser un instrumento acompañante en las demás secciones del tema. 


\section{Trabajo de campo}

Se desarrollaron y se transcribieron los arreglos, teniendo en cuenta las técnicas de la guitarra clásica utilizadas para facilitar la adaptación de cada obra a la interpretación por parte de un solo guitarrista. Seguidamente, se realizó el montaje del repertorio en el grupo de boleros de la Universidad conformado por un percusionista, una cantante y un guitarrista, teniendo como invitado a un trompetista. Finalmente, se produjo un videotutorial ${ }^{2}$ por cada tema, en los cuales se muestra los pasos para su montaje.

\section{Fase analítica}

Se implementó un análisis inductivo, por cuanto permite examinar un fenómeno con miras a descifrar y validar sus significados, generalizando supuestos a partir de los resultados obtenidos. Esto en correspondencia con las metas propuestas, las cuales propendían en su fin último al desarrollo de una propuesta que pudiera ser replicada en entornos similares al aquí trabajado. Es así que se analizó cada arreglo, tomando en consideración las técnicas utilizadas en cada caso, buscando que cada una de las obras sea interpretada por una guitarra solista. Se observaron las decisiones tomadas por los investigadores, las cuales obedecieron a su experiencia interpretativa previa, así como también a sus conocimientos en armonía y composición. La información reunida a partir de los resultados del trabajo de campo permitió entender la pertinencia de la propuesta, así como también determinar las dinámicas y fenómenos que se presentaron alrededor de la misma.

\section{Interpretación musical inteligente}

La inteligencia implica ser capaces de adaptar los recursos disponibles para resolver las problemáticas cotidianas de forma creativa (Gardner, 1993). Es así que en este trabajo se utilizaron los recursos disponibles -que en este caso corresponden a las técnicas de la guitarra clásica- para dar solución al problema de interpretar el género bolero con una sola guitarra. Entre las técnicas propias de la guitarra clásica utilizadas en este estudio se cuentan: ligados ascendentes, descendentes, rectos, apoyaturas, toque tirado y apoyado, pizzicato, melodías a dos notas, armónicos naturales y arrastres. A continuación se describe y justifica el uso de dichos recursos en las obras escogidas.

\section{Toque apoyado}

En la mayoría de estos temas se buscó destacar la melodía en los pasajes escalísticos usando el toque apoyado con los dedos índice y medio, lo que brinda un sonido más potente y percutido a la vez que permite obtener mayor volumen. "El 
dedo de la mano derecha pulsa la cuerda, descansando o deteniendo su impulso en la cuerda inmediata" (Nicola y Pedreira, 2000, p. 14). Esto es propio de la guitarra española y se asemeja más al estilo popular de los boleros. Dicho toque por lo general va acompañado de las notas graves atacadas por el dedo pulgar, por lo que permite que se ejecute la melodía al mismo tiempo con los bajos. Se usa frecuentemente en pasajes donde solo aparece la melodía, lo cual permite destacarla y aumentar su expresividad (Sánchez, 2008).

Para ejemplificar esta técnica se toma el caso de los temas "Quizás, quizás, quizás" y "Sin ti". En la primera obra se ha utilizado el toque apoyado en los compases 1 y 2 de la introducción y el interludio, tal como se muestra en la Figura 1. Este es un pasaje escalístico que requiere virtuosismo y agilidad en su ejecución, en el que se busca un ataque potente sin necesidad que las notas se mantengan sonando. En la segunda obra se utiliza el toque apoyado en toda la línea superior de la introducción ya que permite que sea interpretada con mayor velocidad, además de producir dinamismo en el sonido (Figura 2).

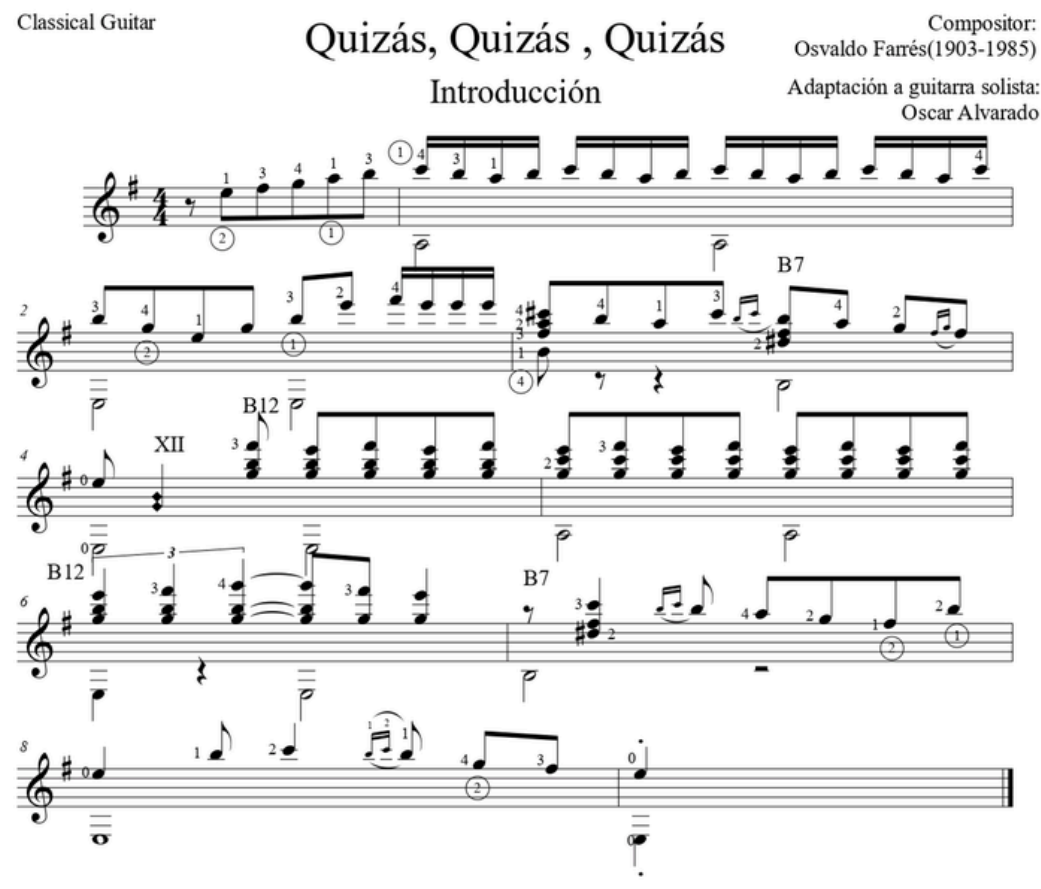

Figura 1. Interludio e introducción de "Quizás, quizás, quizás". Fuente: Elaboración propia. 


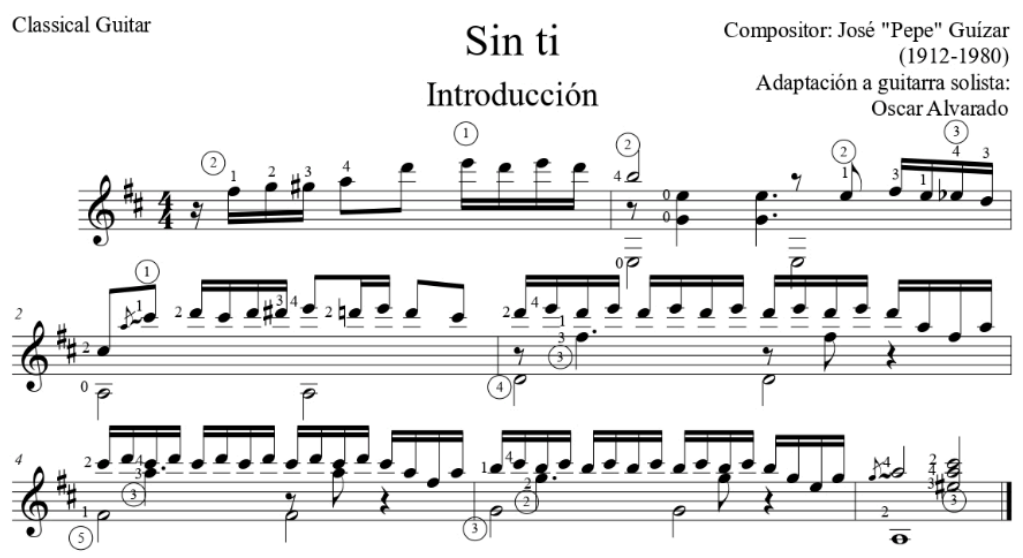

Figura 2. Introducción de "Sin ti". Fuente: Elaboración propia.

\section{Toque tirado}

Se utiliza para lograr un sonido más delicado en los pasajes más lentos, buscando siempre la resonancia de las demás notas del acorde (Carlevaro, 1966), pues con el toque apoyado se apaga la nota de la cuerda superior atacada. Además, es utilizado por lo general en secciones que precisan de una línea melódica serena, ya que posibilita la conservación del equilibrio y la fluidez de la melodía con respecto al acompañamiento (Sánchez, 2008). Su uso en los temas "Y", "Algo contigo", "La barca" y "Sin poderte hablar" se supedita a la reproducción del estilo del bolero.

\section{Pizzicato}

Inicialmente utilizado en instrumentos de arco como el violín y el violonchelo, consiste en producir sonidos asordinados o apagados al pulsar las cuerdas. "Dicho efecto se obtiene colocando el borde lateral de la mano derecha sobre el puente inferior y apoyando el extremo del dedo meñique en la parte inferior de la tapa" (Nicola y Pedreira, 2004, p. 57). En el tema "Sin poderte hablar" se hace uso del pizzicato en la cadencia de la introducción para experimentar con la riqueza tímbrica del instrumento (Figura 3). 


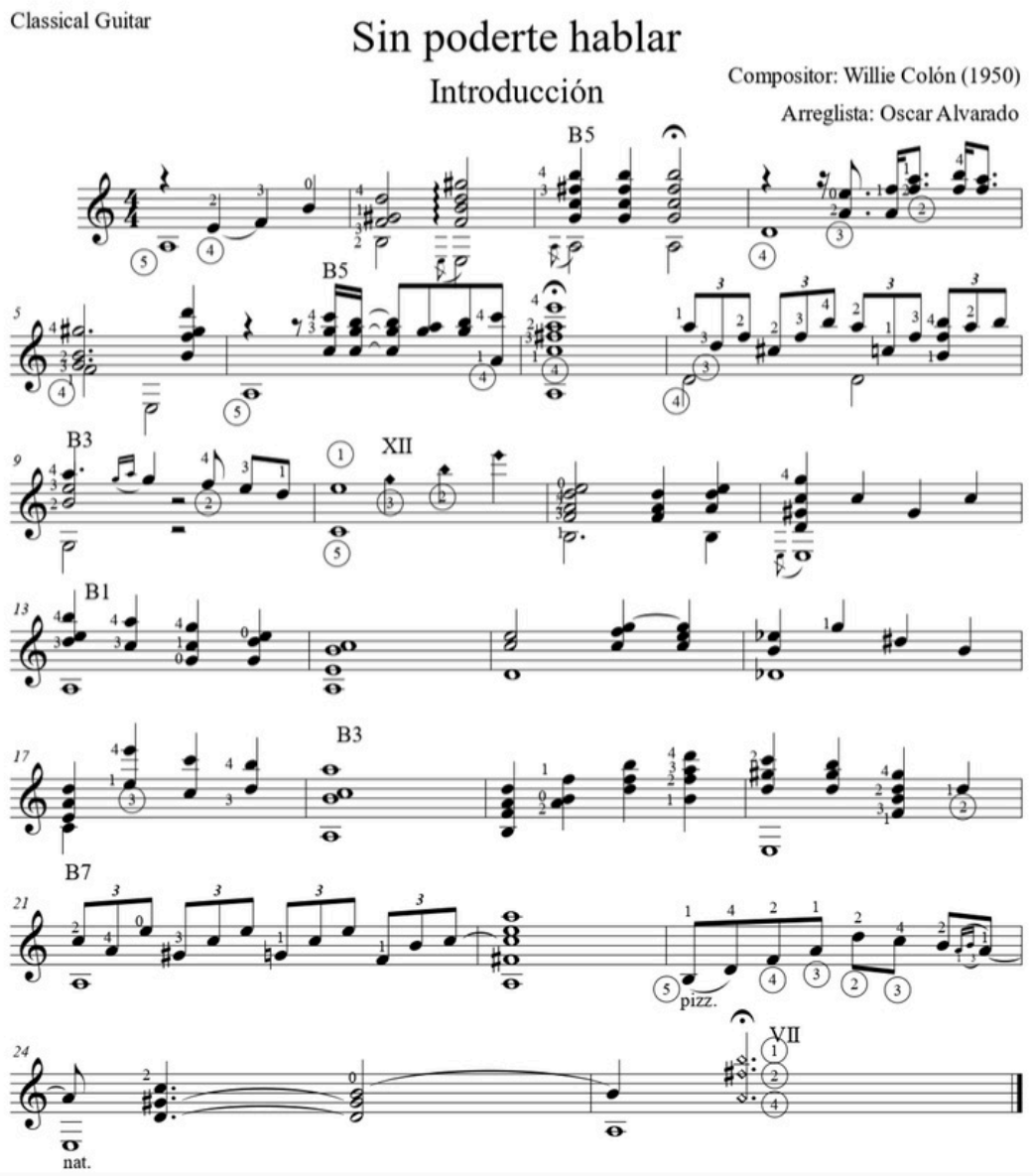

Figura 3. Introducción de "Sin poderte hablar". Fuente: Elaboración propia.

\section{Armónicos naturales}

"Se producen tocando una cuerda con la yema de un dedo, sin apretarla, encima de las divisiones de su longitud, que algunas corresponden con la de los trastes" (Aguado, 1843, p. 42). En "Sin poderte hablar" (ver Figura 3) se ejecutan los armónicos únicamente con la mano derecha, colocando el dedo índice sutilmente sobre el traste 12 y con el dedo anular se pulsa la cuerda. De esta forma se mantiene resonando el bajo pulsado con la mano izquierda.

Además, los armónicos brindan posibilidades tímbricas que posibilitan una 
variedad de sonidos, haciendo más interesante la obra. Es el caso del tema "Quizás, quizás, quizás", en el cual al término de la primera frase, la melodía se ve complementada con los armónicos naturales, lo cual se muestra claramente en la Figura 1.

\section{Melodías a dos notas}

En algunas obras y estudios del repertorio clásico se manejan melodías a dos notas (terceras, sextas, entre otras). "Una de las ventajas que ofrece la guitarra es que una misma posición de dedos de la mano izquierda sirve para ejecutar el mismo intervalo en todas las cuerdas" (Aguado, 1843, p. 39), con lo que se facilita alcanzar una digitación más fluida. En "La barca" (figura 4) se hace visible el uso de esta técnica, tal como se describe más adelante.

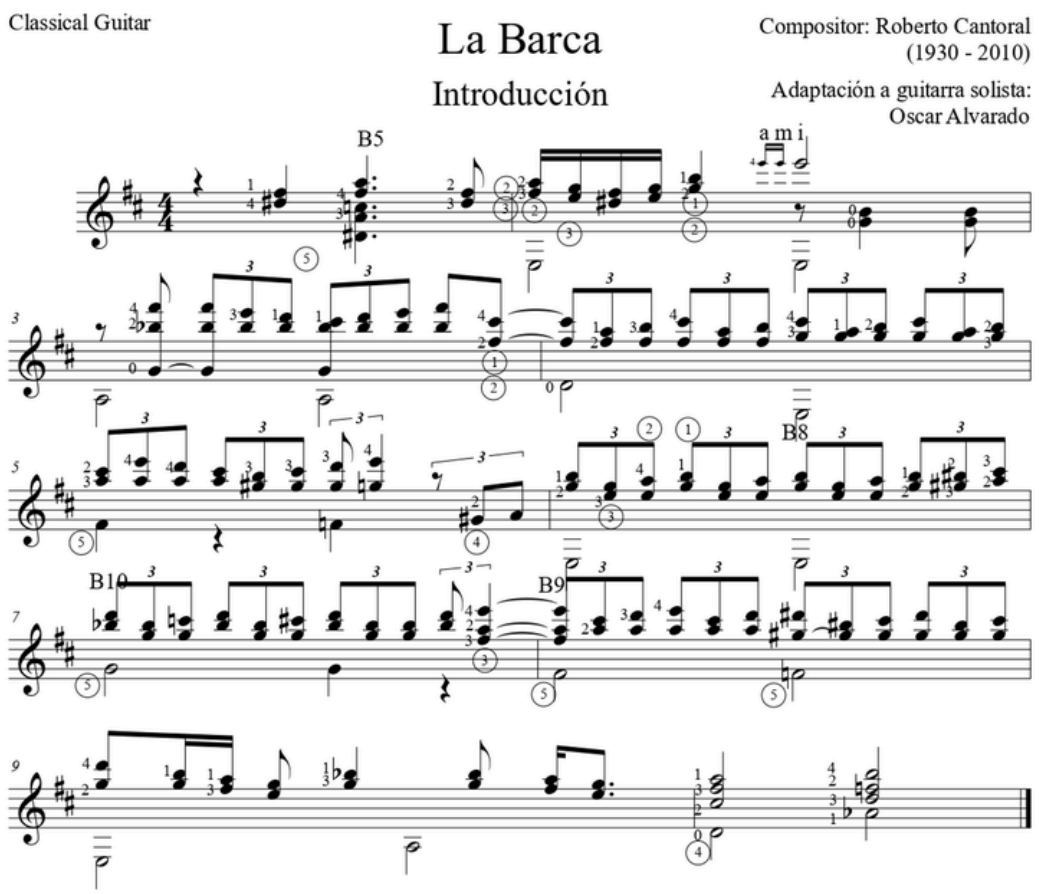

Figura 4. Introducción de "La barca". Fuente: Elaboración propia. 


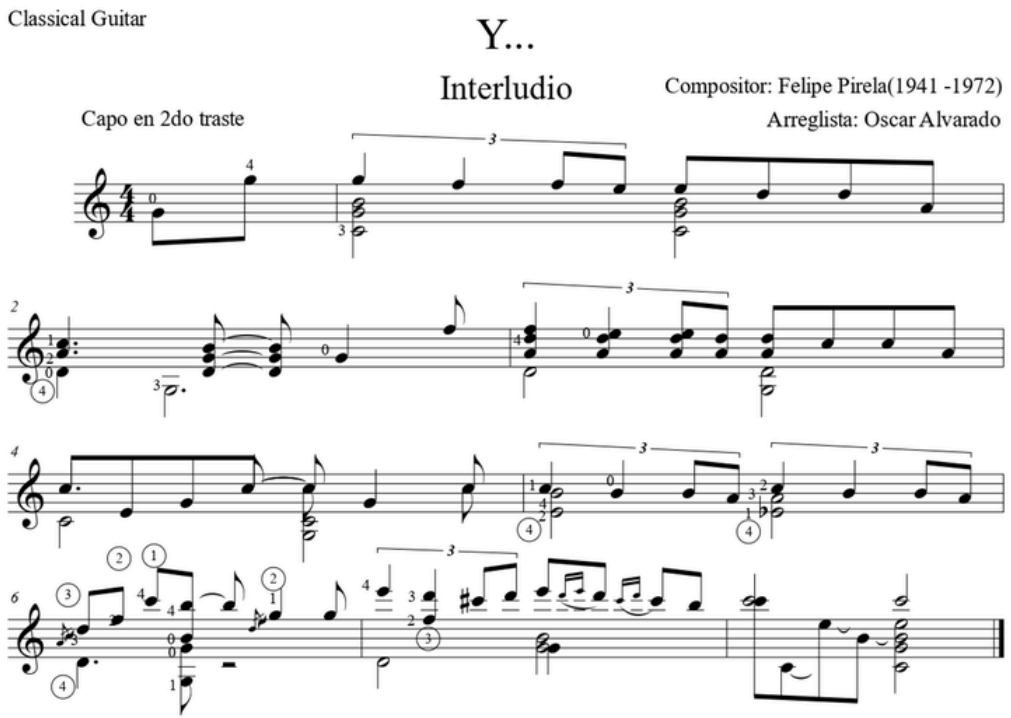

Figura 5. Interludio de "Y". Fuente: Elaboración propia.

\section{Ligados y apoyaturas}

Según Dionisio Aguado (1843), en el ligado "la mano izquierda ejecuta dos, tres o cuatro notas, subiendo o bajando, sin que la mano derecha haya pulsado más que la primera" (p. 29), mientras que la apoyatura consiste en "una nota muy corta que toma su valor de la nota siguiente" (p. 31). Ambas técnicas permiten una mayor velocidad, ya que con un solo ataque se producen más sonidos. Es así que en los temas "Y" (figura 5), "Quizás, quizás, quizás", "Algo contigo" y "Sin poderte hablar", los ligados y apoyaturas utilizados en sus introducciones e interludios pretenden mantener el estilo y la sonoridad del trío de boleros.

\section{Arrastre}

Para ejecutar este recurso el dedo pisa la primera nota y recorre los trastes hacia la otra ejerciendo la presión justa, de forma que se escuchen las cromas entre ellas dos (Rodríguez Arenas, 1964), sublime efecto sonoro que involucra ir de una nota a otra en la misma cuerda pasando cromáticamente por otras notas es propio del estilo bolero. En el caso de los temas "Y", "Algo contigo" y "Sin ti", el empleo de esta técnica además permite que la digitación sea más fluida y cómoda para la mano izquierda. 


\section{Barra o ceja}

El dedo 1 se despliega sobre todas las cuerdas mientras se descansa la última falange del dedo pulgar sobre la mitad del mango, por lo que el punto de apoyo sube un poco más de lo habitual sin que actúe la primera falange (Roch, 1921). Por su parte, la media barra se ejecuta de igual manera pero extendiendo el dedo sobre 2, 3, 4 o 5 cuerdas, según sea necesario. Para efectos de los arreglos aquí realizados, tanto la barra como la media barra se utilizan buscando combinar melodía y acompañamiento de la forma más efectiva posible.

\section{Obras arregladas: seis casos de estudio}

Habiendo descrito las principales técnicas utilizadas se hace pertinente analizar su aplicación y combinación en cada una de las obras arregladas. Cabe resaltar que al elaborar los arreglos se comenzó por transcribir la melodía principal, a la que posteriormente se le agregaron algunas notas de adorno, generalmente intervalos de terceras y sextas. Esto contribuyó a que el sonido se aproximara al de un requinto, instrumento que suele ejecutar esta parte del tema dentro del ensamble de trío o grupo musical. Luego se hizo un análisis de la armonía de la canción conjugándola con la melodía, siempre teniendo en cuenta la célula rítmica del bolero. Aquí se buscaron las mejores posibilidades de digitación que permitan explotar los recursos de la guitarra de forma que sea interpretada por un mismo guitarrista.

\section{La barca}

Tanto en la introducción como en el interludio se ejecuta la melodía a dos notas. En el segundo y tercer tiempo del primer compás se encuentran terceras menores en la primera y la segunda cuerda. El primer intervalo de tercera D\# y F\# se ejecuta en estas cuerdas buscando llegar al acorde disminuido (D\#07) desde la quinta cuerda. Este contiene en las dos primeras cuerdas el segundo intervalo de tercera menor (F\# y A). De este modo el cambio de posición se ejecuta con mayor naturalidad. Sin embargo, como expresa Aguado (1843), esta ventaja no se cumple entre la segunda y la tercera cuerda por lo que, en este caso, los mismos intervalos realizados anteriormente ahora se ejecutan en segunda y tercera cuerda para llegar de forma orgánica al acorde de Em, y posteriormente tocar la nota $\mathrm{E}$ en el XII traste de la primera cuerda.

Por otra parte, se aprecia repetidamente el uso de medias barras, lo que permite llevar las melodías a dos notas al tiempo con los bajos. En el segundo compás, al llegar al acorde de Em se realiza un trémolo sobre una nota aguda del mismo. Esto usualmente sería ejecutado por un guitarrista puntero utilizando un pick o plector, pulsándolo de arriba hacia abajo simultáneamente. Para el caso particular de este 
arreglo, la nota es atacada en el orden habitual del trémulo en la guitarra (anular, medio, índice), pues se hace al mismo tiempo que el bajo y posteriormente se toca su acorde. De esta forma se logra sacar el máximo provecho a la mano derecha, demostrando su virtuosismo.

En este tema, la introducción y el interludio son similares, presentando una única diferencia en su cadencia. Es así que, mientras en casi todo el interludio la melodía se desarrolla a dos notas, en los compases octavo y noveno se interpreta la cadencia por medio del toque apoyado, lo que permite resaltar su carácter escalístico y concluir el tema de forma contundente.

\section{Y}

En el segundo compás de la introducción se aprecia cómo la nota $G$ de la melodía se toca con dedo 2 en el III traste en la primera cuerda. Luego, buscando llegar al mismo $G$ pero esta vez en la segunda cuerda, se cambia el dedo 1 del bajo para ejecutar un arrastre, desde el III traste de la segunda cuerda al VIII traste, con lo que se llega fácilmente a un E agudo del XII traste con el dedo 4. Este arrastre permite tocar una misma nota en dos cuerdas diferentes para abordar una nota más cercana, sacrificando un instante del valor del bajo para no cortar la melodía. El mismo procedimiento se aprecia en el sexto compás, esta vez involucrando otras notas y acordes pero con el mismo objetivo de digitación.

Además, en la segunda mitad del tercer compás se hacen unas apoyaturas a las notas D y C de la melodía para adornarlas. Cabe resaltar aquí la influencia que tiene el gusto personal de los investigadores al momento de elegir ciertas técnicas. Tal como afirma Emilio Pujol (1960), los sonidos le otorgan sentido a la sensibilidad musical, no solo del intérprete sino también del que la escucha y visibiliza sus subjetividades, lo cual no aparece definido por la razón o los cálculos.

Por otra parte, en el interludio de este tema la melodía siempre va acompañada por otro intervalo. Es por esto que se tocan dichas notas con el dedo índice y medio o medio y anular al mismo tiempo, como se trabajaría un estudio de terceras o sextas de algún compositor de la escuela de la guitarra clásica. Esto se hace con el fin de imitar el efecto que se logra en un trío tradicional de bolero cuando una de las guitarras acompañantes interpreta la melodía al mismo tiempo que la guitarra requinto, produciendo un sonido más lleno y colorido.

\section{Algo contigo}

En la mayor parte de esta obra, con excepción de los ligados ascendentes y descendentes, encontramos que se utiliza un toque tirado de la mano derecha y de esta manera realizamos la melodía sin perder ninguna nota de los acordes, generando una atmósfera muy agradable al oído. En la introducción se aprecia el 
uso de ligados descendentes en el primer y en el segundo compás. En el tercer tiempo se hace una apoyatura para cambiar de bajo del $\mathrm{G}$ al F en el tercer compás, dándole un poco más de carácter a ese bajo y resaltando la llegada.

En el sexto compás se hace un arrastre de C a D en la quinta cuerda para lograr que suenen al tiempo el D de la cuarta cuerda al aire y el D de la quinta cuerda, seguido de una apoyatura en el cuarto tiempo para embellecer esta nota.

En el interludio en el tercer compás se hace una barra en el VII traste, de esta manera se logra el acorde y a su vez la nota B en la melodía. De la misma manera se hace una media barra en el quinto compás, teniendo el acorde y la nota $\mathrm{F}$ de la melodía.

\section{Sin poderte hablar}

Se comienza haciendo una introducción ad libitum siguiendo siempre al cantante y buscando la mayor resonancia de notas posible, para lo cual se utiliza un toque tirado de la mano derecha con el que no se apaga ninguna nota y brinda un sonido más dulce. En el tercer compás los acordes se ejecutan con la yema del dedo pulgar de la mano derecha, con el fin de generar un sonido opaco. Por otro lado, en el compás 21 se utiliza el pizzicato generando un contraste a la interpretación a comparación del resto de la pieza.

\section{Quizás, quizás, quizás}

La primera parte de la introducción es un pasaje de carácter escalístico para el cual se utiliza el toque apoyado de la mano derecha. En el tercer compás se utiliza una apoyatura sobre la barra, la cual se debe hacer por la nota del bajo y en la última corchea de este mismo compás también se hace apoyatura a esta nota. En el cuarto compás se aprecia cómo se completa el acorde con armónicos naturales y luego se hace una barra en el traste XII hasta el sexto compás, con el fin de poder llevar la melodía y la armonía al mismo tiempo. Finalmente, en los últimos compases se utilizan algunas apoyaturas para embellecer las notas.

\section{Sin ti}

Debido al virtuosismo requerido, la introducción se ejecuta con toque apoyado, tal como lo hacen los guitarristas que componen los tríos de boleros tradicionales. En el segundo y sexto compás se hacen arrastres para embellecer la nota y para llevar a cabo el cambio de posición. Aquí, la dificultad radica en ejercer el toque apoyado sin que el dedo pulgar descanse en la sexta cuerda (como suele hacerse), ya que debe tocar los bajos y algunas notas del acorde. 


\section{Conclusiones}

Teniendo en cuenta todo lo descrito anteriormente, con el desarrollo de este proyecto se logró impactar en tres aspectos principales: el componente artístico con la grabación de los seis temas propuestos dirigido a toda clase de público, el componente académico por medio del diseño de un libro de partituras con los arreglos realizados y este artículo de investigación, y el componente pedagógico a través de la producción y difusión de seis videotutoriales en la plataforma institucional.

Además, a partir del análisis del proceso creativo y de los productos obtenidos, se ha logrado arribar a las siguientes conclusiones:

- Las técnicas aplicadas permiten al guitarrista ejecutar con menor dificultad y mayor agilidad, demostrando el virtuosismo que cautiva al público y permitiendo alcanzar la sonoridad brillante que caracteriza el estilo abordado.

- El estudio del repertorio popular en un ambiente académico se hace relevante por cuanto provee habilidades creativas e interpretativas y otorga sentido a sus aprendizajes al contextualizarse en sus realidades.

- La interconexión entre los roles del músico actual -docente, creador e intérprete- es indispensable para el desarrollo de estudios que enriquezcan el legado musical de los géneros populares y de los pueblos que los producen.

Finalmente, esta investigación ha permitido sistematizar el uso de técnicas propias de la guitarra clásica para interpretar el género bolero, situación que no es precisamente novedosa pues se conoce que es una práctica bastante generalizada que se lleva a cabo de forma empírica. Sin embargo, tal como se evidenció en la búsqueda de antecedentes, no se cuenta con referentes de investigación que expongan el proceso de forma rigurosa. Es así que se insta a los lectores a continuar ahondando en este tema, investigando la adaptación de estas y otras técnicas en la interpretación de otros géneros populares y modernos.

\section{Notas}

1. Oscar Julián Alvarado Laurín: https://orcid.org/0000-0001-7562-1424

2. Oscar Alejandro Beltrán Sánchez: https://orcid.org/0000-0002-8753-6865

3. La barca: https://www.youtube.com/watch?v=wR3bRXnXtAM\&t=2s; Quizás, Quirás, quizás: https://www.youtube.com/watch?v=Pcwg6FdY7O4\&t=180s; Sin poderte hablar: https:// www.youtube.com/watch? ${ }_{\mathrm{v}}=\mathrm{qKYPT}-\mathrm{pIRkc \& t}=15 \mathrm{~s}$, Algo contigo: https://www.youtube.com/ 
watch?v $=x y C o g X U o O i c \& t=46 s ;$ Sin ti: https://youtu.be/oTsUOZeg2w8; Y: https://youtu.be/ e4BnZj0bgR4

Las partituras de los arreglos están reunidas en Alvarado, O. (2019) Seis cuerdas el bolero. Inédito Disponible en: https://drive.google.com/file/d/1jyzobqS2s1AmbeYNVsxJwRu_RKF4GoBt/ view?usp=sharing

\section{Referencias}

Aguado, D. (1843). Nuevo método para guitarra. París: Schonenberger.

Arzubiaga, J. P. (2007). Apuntes sobre el bolero: desde la esclavitud africana hasta la globalización. Revista de ciencias sociales, 19, 95-117.

Barrera, A. M. (2015). Plan rectoral 2015-2019 de la Universidad de Cundinamarca generación siglo XXI. Recuperado del sitio de la Universidad de Cundinamarca: https:/ / www.ucundinamarca.edu.co/index.php/universidad/planeacion-institucional/planrectoral-2015-2019

Buil Tercero, P., y Hormigos Ruiz, J. (2016). Nuevas formas de distribución de la música popular en la cultura contemporánea. Revista de Ciencias Sociales, 4(1), 48-57. doi: $10.17502 /$ m.rcs.v4i1.103

Carlevaro, A. (1966). Serie didáctica para guitarra. Cuaderno número 1. Escalas diatónicas. Buenos Aires: Barry Editorial.

Cerda, H. (2001). El proyecto de aula. Bogotá: Magisterio.

García, J. J. (2016). ¿Son o salsa? Agenda cultural Alma Mater, 233, 10-12. Recuperado de https://revistas.udea.edu.co/index.php/almamater/article/view/323454

Gardner, H. (1993). Multiple intelligences: the theory and practice. New York: Basic Books.

Londoño, M. E., y Tobón, A. (2004). Bandola tiple y guitarra: de las fiestas populares a la música de cámara. Artes, la revista, 4(7), 44-68. Recuperado de https://revistas.udea. edu.co/index.php/artesudea/article/view/22847

Nicola, I., y Pedreira, M. (2000). Método de guitarra. Primer Curso. La Habana: Atril Ediciones.

Nicola, I., y Pedreira, M. (2004). Método de guitarra. Cuarto Curso. La Habana: Atril Ediciones.

Pagano, C. (1989). El bolero en Colombia: un viejo amor. Revista Universidad Nacional, 20, 37-48. Recuperado de https://revistas.unal.edu.co/index.php/revistaun/article/ view/12004 
Epistemus - volumen 8 - número 1 (Julio de 2020)

DOI: https://doi.org/10.24215/18530494e017

Pujol, E. (1960). El dilema del sonido en la guitarra. Buenos Aires: Ricordi Americana.

Rodríguez Arenas, M. (1964). La escuela de la guitarra. Libro III. Buenos Aires: Ricordi Americana.

Roch, P. (1921). Método moderno para guitarra (Escuela Tárrega). New York: G. Schirmer Inc.

Sánchez, D. F. (2008). Análisis técnico e interpretativo de la Cadenza del concierto para Guitarra y Pequeña Orquesta de Heitor Villalobos (Tesis de grado). Pontificia Universidad Javeriana, Colombia.

Thoms, W. J. (1846). Folklore. Athenaeum, 982. Recuperado de Link correcto: https:/ / www.jstor.org/stable/41306605?seq=1

Villegas, P. (2016). Estrategia y táctica para revitalizar el bolero. Revista Cubana De Antropología Sociocultural, (7)8, 89-104. Recuperado de http://www.revista-batey.com/index. $\mathrm{php} / \mathrm{batey} /$ article/view/136/89

Libro de partituras https://drive.google.com/file/d/1jyzobqS2s1AmbeYNVsxJwRu_ RKF4GoBt/view?usp=sharing 